\title{
The Influence of Load on Risk-based Small-Disturbance Security Profile of a Power System
}

DOI:

10.1109/TPWRS.2017.2693502

\section{Document Version}

Accepted author manuscript

Link to publication record in Manchester Research Explorer

\section{Citation for published version (APA):}

Hasan, K., Preece, R., \& Milanovic, J. (2017). The Influence of Load on Risk-based Small-Disturbance Security Profile of a Power System. IEEE Transactions on Power Systems, PP(99).

https://doi.org/10.1109/TPWRS.2017.2693502

\section{Published in:}

IEEE Transactions on Power Systems

\section{Citing this paper}

Please note that where the full-text provided on Manchester Research Explorer is the Author Accepted Manuscript or Proof version this may differ from the final Published version. If citing, it is advised that you check and use the publisher's definitive version.

\section{General rights}

Copyright and moral rights for the publications made accessible in the Research Explorer are retained by the authors and/or other copyright owners and it is a condition of accessing publications that users recognise and abide by the legal requirements associated with these rights.

\section{Takedown policy}

If you believe that this document breaches copyright please refer to the University of Manchester's Takedown Procedures [http://man.ac.uk/04Y6Bo] or contact uml.scholarlycommunications@manchester.ac.uk providing relevant details, so we can investigate your claim.

\section{OPEN ACCESS}




\title{
The Influence of Load on Risk-based Small- Disturbance Security Profile of a Power System
}

\author{
Kazi N. Hasan, Member, IEEE, Robin Preece, Member, IEEE, and Jovica V. Milanović, Fellow, IEEE
}

\begin{abstract}
This paper establishes a generic severity function that can be used to produce power system security risk profiles. It is illustrated by analyzing the impacts of system load attributes on the small-disturbance rotor angle stability of a power system. The load attributes contributing to the oscillatory modes can be considered as inherent uncertain variables within power systems and include load power variations, load composition, and load model parameters. Uncertainty in the renewable power generation is also incorporated in the probabilistic modelling and risk assessment to demonstrate the flexibility of the approach. A novel approach is proposed to select the severity functions to logically represent small-disturbance security margin. The risk profile of a power system has been presented considering the probability density functions (pdfs) of power system critical modal damping and a selected set of severity functions. The analysis techniques developed are illustrated with a modified version of the 68-bus NETS-NYPS power system with a high amount of renewable power penetration. The relative importance of the load attributes and the impact of these attributes on stability boundaries has been identified at varying risk levels with respect to their contribution to small-disturbance stability.
\end{abstract}

Index Terms-Load modelling, power system analysis computing, probabilistic assessment, risk analysis, small-signal stability, uncertainty.

\section{INTRODUCTION}

$\mathrm{L}$ IBERALIZATION of the electricity markets, renewable

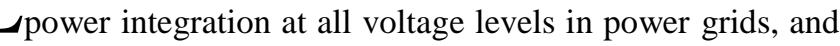
new types of loads bring power system operation to a new paradigm where the system is operating closer to the limits of security. Deregulation of the generation, transmission and distribution leads to more unpredictability of dispatch and consumption patterns, whereas increased distributed energy resources add further to uncertainty of supply. Hence, the inherent uncertainty in modern power system operation reduces the flexibility in decision making and subsequently increases the risk of instability [1].

Limitations of deterministic approaches to handle uncertain situations have been discussed in previous research with an emphasis on the need for risk-based security assessment of power systems [2-5]. A risk-based framework does not only

This work is supported by the EPSRC, UK, through the Supergen+ for HubNet project (grant number: EP/M015025/1).

The authors are with the School of Electrical and Electronic Engineering, The University of Manchester, Manchester, M60 1QD, UK. (email: kazi.hasan@manchester.ac.uk, robin.preece@manchester.ac.uk, jovica.mila novic@manchester.ac.uk) provide the probability of occurrence of an unexpected event but also quantifies the severity of the consequence of the occurrence of that event. Subsequently, it provides a riskprofile of the system that can be used to comprehend the varying risk levels of different operating conditions.

Power system risk assessment can be divided into two basic aspects, which are adequacy and security-based risk profiling [1]. They correspond to static and dynamic states of a power system, respectively. This study considers the security-based risk assessment, which conforms to the operational time-frame and therefore may not be suitable for a long term planning horizons. A number of applications of security-based risk evaluation have been demonstrated in several areas such as voltage stability [2], overload and low voltage security [3], frequency stability [4] and transient stability [5]. However, the risk assessment of small-disturbance stability has received less attention within power system research. The $p d f$ of the real part of the critical system state matrix eigenvalues (to determine the probabilistic instability) has been considered as normally distributed in [6], which is often not a correct assumption [7]. A small number of attempts to perform risk assessment of small-disturbance instability have been presented in [8-10]. However, these works solely focus on the probability of instability, ignoring the severity of the corresponding instability, and although very valuable, are not truly risk assessments. The probability is only a part of the risk assessment, as the risk is a combination of the likelihood (probability) of occurance of an event and the consequence (severity) of that event. Small-disturbance instability analysis presented in [11] considers a discrete risk matrix, which ranks the severity of risk as low, medium and severe. This does not completely reflect the continuous extent of the risk. The severity functions presented in [7] are limited in the following ways. The ramp and quadratic severity functions do not suitably distinguish between a marginally stable and a marginally unstable case. For example, these functions would provide almost equal severity values to cases where oscillation damping is equal to $-0.001 \mathrm{~s}^{-1}$ and $0.001 \mathrm{~s}^{-1}$, despite the fact that the latter case is unstable and more severe. The step function used, on the other hand, is limited by the assumption that it considers all stable cases equally and all unstable cases equally, which does not capture the true extent of severity. For example, it would not capture the difference between oscillation damping $0.01 \mathrm{~s}^{-1}$ and $0.1 \mathrm{~s}^{-1}$, although clearly the latter is more severe as unstable oscillations would grow more rapidly and less time would be available for corrective control 
actions.

Present research into risk-based small-disturbance security assessment is lacking appropriate severity functions, which limits the capability to properly quantify risk profiles. True cost-based stability severity functions do not exist and cannot be obtained without significant further work to establish the cost of maintaining stability margins for system operators. To overcome the limitations of previously discussed severity functions, a multi-slope severity function is proposed in this paper to appropriately weight the stable, marginally stable and unstable regions of the damping of oscillations in the risk assessment. The semi-continuous severity function presented in this paper clearly distinguishes between stable and unstable situations and appropriately weights the oscillation damping according to the eigenvalue position in the complex plane. The proposed severity function also possesses relative indicative signals when the system approaches closer to instability. This will be discussed further in Section II.

Power system-load interaction is considered as a critical source of rotor angle oscillatory instability [12, 13]. Many different load attributes contribute to the system oscillations. Uncertainty in load has a high impact on power system security [14]. The proportion of dynamic load has a considerable impact on oscillation damping [15]. Load characteristics also have a major effect on the stability of the interarea modes [16]. Earlier works identified system loads as the most influential elements within a power system network with respect to their contribution to the small-disturbance stability $[17,18]$. The risk profile of a power system can be largely influenced by system load attributes. Though the significance of load attributes on power system stability is well documented in the literature [12-16], there is no work, to the best of our knowledge, which explicitly assesses the influence of load attributes on system security risk.

This paper quantifies the risk incurred by a power system due to load attributes such as load power variations, load composition, and load model parameters. The paper makes the following key novel contributions:

- Proposition of a coherent and appropriate risk severity function to represent the relative severity of power system small-disturbance marginal stability and instability.

- Quantification of power system risk-level corresponding to system load attributes - highlighting their relative contribution to small-disturbance security.

- Identification of the probabilistic security boundary for different values of load attributes.

- Thorough sensitivity analysis of system risk as affected by the parameters of the severity functions.

The small-disturbance security based risk profile facilitates the identification of the level of risk at different operating conditions and for a range of system parameters. The level of change in risk due to the changes in system parameters helps in identifying the most influential system parameters. The risk profile presents a clear visual representation of high-risk operating conditions susceptible to power system instability as well as secure stable zones of operation.

\section{QUANTIFICATION OF RISK}

Risk expresses the effect of loss, or other adverse circumstances. Risk can be quantified as the product of the probability of a detrimental event and the severity of that event. These concepts of risk are event-based and are deterministic by nature. Risk can be defined as a probabilistic phenomenon and quantified through the use of continuous severity functions. In such a case, risk is an expectation of severity summed across the product of all outcome probabilities and their corresponding severity [2]. A risk index can be a single number (for one study parameter with respect to a certain threshold), a line (for two study parameters), a surface (for three) or a hypersurface (for more than three).

\section{A. Value at Risk (VaR)}

Value at Risk (VaR) is the most widely used risk measure. It can identify the worst case scenario (i.e. maximum expected loss) of a portfolio within a specified degree of confidence.

In the power system stability measures, at a given confidence level $\alpha \in(0,1)$, the $\mathrm{VaR}$ of the portfolio at the confidence level $\alpha$ is given by the smallest number $l$ such that the probability that the $\operatorname{loss} L$ exceeds $l$ is at most 1$\alpha$. Mathematically, if $L$ is the amount of a portfolio, then $\operatorname{VaR}(L)$ is the level $\alpha$-quantile, i.e.

$$
\operatorname{VaR}_{\alpha}(L)=\inf \{l \epsilon R: P(L>1) \leq 1-\alpha\}
$$

The VaR limit enables the identification of the maximum threshold values of power system parameters (such as individual load attributes) corresponding to the point at which the system crosses the stability margin.

\section{B. Severity Functions}

A severity function (SF) quantifies the severity of the network condition in terms of its performance indicators [3]. It is a common practice in the quantification of risk to express the SF in terms of economic worth. As there is currently no known information that can be used to put a price on power system stability indicators, this study considers only the technical indicators in the representation of the SFs. This is a standard approach in power system stability risk assessment.

1) Properties of the severity function

The essential criteria for choosing a severity function (SF) in risk-based security assessment have been documented in [2]. The SF should; i) reflect the consequence of the contingency; ii) be physically understandable; iii) be tied to deterministic criteria; iv) be relatively simple; v) reflect relative severity, and vi) measure the extent of a violation.

Continuous SFs have been considered earlier in power system security [2, 3, 5] and small-disturbance stability assessment [7]. A single ramp-function has been considered in some previous research as an SF for assessing the risk of critical clearing time (transient stability analysis) and overload and low voltage phenomenon (voltage stability studies) [3, 5]. Both step- and a ramp-function based SFs have been described for low voltage, overload and voltage instability around their corresponding security boundary [2]. Further, step-, ramp- and quadratic-function based SFs have been proposed to assess the risk of small-disturbance stability [7]. 
The ramp, step and quadratic-functions have been implemented as over-simplified severity functions. The violation of the stability limit or system collapse does not get enough importance in the linear-and quadratic functions as the value of SF remain almost the same just before and after the stability line (y-axis). These SFs violate the criteria i), iii), v) and vi). Furthermore, when the SF approaches instability there is no indication in the step function that it is close to the stability limit. This is a violation of the criteria ii) and v). The slope of the ramp-function, on the other hand, in the stable region and unstable region is the same, despite the fact that the later state is more severe. This is against the criteria v) and vi). Moreover, in the step-function, the severity remains the same throughout the whole unstable region, though it is clearly more severe to penetrate deeper into the unstable region. This is a violation of criteria ii) and $v$ ).

To overcome the limitations of previously mentioned SFs, a multi-slope SF is proposed as shown in Fig. 1. The proposed SF eliminates the limitations as discussed above and conforms to the criteria mentioned for the SF of the risk-based security assessment. Its application in the assessment of risk for smalldisturbance stability exhibits following severity properties:

(a) It weights the 'secure' stable region lightly with respect to the unstable region with lower slope of the curve. Also, the weight increases as it moves towards the marginal stability line. This reflects higher severity as the system approaches instability. (Conforms to the criteria iv) and v)).

(b) The (vertical line) y-axis is an essential part of the severity representation as it distinguishes the stable and unstable region. This brings the unstable severity curve at a higher value in y-axis to reflect the instability effect. A high yintercept of the instability curve weights the unstable scenario appropriately. (Criteria i) - vi)).

If the y-intercept for stable and unstable region is the same, the curve becomes a linear ramp function. This curve is violating the criteria i), ii), iii), v), and vi) of the SF as mentioned above. This issue will be further explained with illustrative results (Section V.D) in the paper.

(c) High slope of the ramp-function in the instability region can reflect the extreme severity of the small-disturbance stability. (Criteria iv), v) and vi)).

2) Guidelines of parameters selection of the severity function

It has been discussed (and will be later illustrated) that the proposed shape of the continuous SF is suitable for stabilitybased risk analysis. The next step is to identify appropriate parameters. One version of the continuous SF is shown as the solid line in Fig. 1. The parameters which describes the proposed continuous SF are: $\mathrm{X}$-intercept $x^{y 0}$, the slope of the curve in stable region $k_{1}$, Y-intercepts $y_{1}^{x o}, y_{2}^{x o}$, and the slope of the curve in unstable region $k_{2}$.

For small-disturbance stability analysis, the $\mathrm{X}$-intercept $\left(x^{y 0}\right)$ is most appropriately calculated from the settling time of the oscillations, as presented by (2) [7].

$$
\begin{aligned}
& T_{s}=\ln (\text { tolerance/damping }) \\
& \text { or, } x^{y 0}=\ln (\text { tolerance }) / T_{s}
\end{aligned}
$$

Let the slope of the curve in stable region be $k_{l}$. A higher value of $k_{l}$ will assign more weight to system severity as it approaches marginal stability. The equation of the curve in the stable region can be represented simply as in (3).

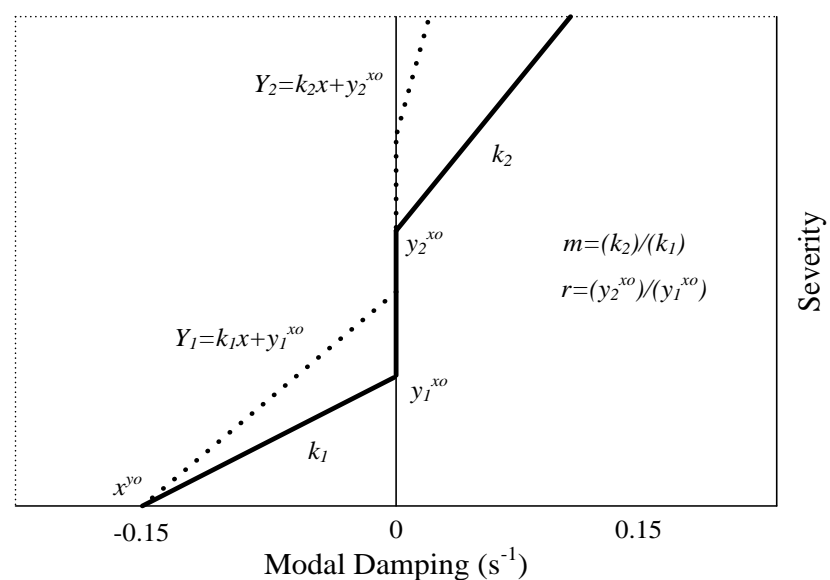

Fig. 1. Continuous severity functions for risk calculation considering smalldisturbance stability.

$$
Y_{1}=k_{1} x+y_{1}^{x o}
$$

The Y-intercept of the curve in the stable region is $\left(y_{1}^{x o}\right)$. Now, a discontinuity is introduced so that the curve of unstable region will have a different $\mathrm{Y}$-intercept $\left(y_{2}^{x o}\right)$, which represents how much more severe a marginally unstable case is than a marginally stable scenario. The Y-intercept of stable and unstable region is related through the intercept ratio $r$ as presented in (4).

$$
r=y_{2}^{x o} / y_{1}^{x o}
$$

Let the slope of the curve in unstable region be $k_{2}$. The equation for the curve in unstable region can be denoted as in (5).

$$
Y_{2}=k_{2} x+y_{2}^{x o}
$$

The slope $k_{2}$ can also be represented by the slope relative to $k_{1}$, as shown in (6).

$$
m=k_{2} / k_{1}
$$

This is representing the relative severity of the security violation as it penetrates to the unstable region, and is termed the severity multiplier $m$.

The values of $r$ and $m$ will depend on the choice of individual operating utility company in conformance to their severity (and reliability) criteria. Also, these parameters can have different values to weight different loads or parts of the network based on the priority of the connected customers. For example, areas with high priority customers such as hospitals, fire services and other emergency services can have a high value of these parameters, and hence showing a high value for security-based risk profile.

If $r=1$ and $m=1$, then (5) becomes the same as (3), presenting a standard ramp function. As has been discussed already, this selection of SF does not appropriately differentiate the stable and unstable cases. Hence, other sets of values for $r$ and $m$ need to be considered. Section V.E presents a detailed sensitivity analysis of the SF parameters. Depending on the values of $r$ and $m$, the system risk profile may be accentuated in different ways. This will be explored further within this paper. An alternative SF has also been illustrated by the dotted lines in Fig. 1. Illustrative results with an exponential increase of severity in the unstable region will 
also be discussed in Section V.D.

\section{3) Risk calculation}

The total system risk can be calculated by integrating the area of probability (probability density function, $P(\sigma)$ ) multiplied by the area of severity (continuous SF, $S(\sigma)$ ). In case of small-disturbance stability, both probability and severity are functions of critical mode damping, $\sigma$. Hence, the risk can be represented as in (7).

$$
\text { Risk }=\int P(\sigma) S(\sigma) d \sigma
$$

Many numerical integration techniques can be used to calculate the total risk. In this work, the trapezoidal method has been used.

\section{SySTEM-LOAD INTERACTION AND LOAD ATTRIBUTES}

The changes in power system load are either almost continuous small variation due to regular customer activities or more, more rare, sudden disturbances caused by abrupt load changes. The latter are typically the subject of transient, large disturbance voltage and frequency stability studies, hence in this study, only the small (almost continuous) load disturbances/variations are considered. Fig. 2(a) shows the system-load interaction in a power system, whose details are available in [12, 13]. More discussion on the interaction of non-linear dynamic loads with power system, industry practice on load modelling and measurement based load modelling approach can be found in [19-23]. Load characteristics have a high influence on the small-disturbance stability of a power system [16]. Inadequacy of load modelling has been identified as one of the most important challenges for risk-based and probabilistic planning and operational studies [14].

\section{A. Load Attributes}

Specific aspects of loads have been previously identified as contributing towards the small-disturbance instability of power systems. These load attributes are load variability, system loading, load composition and load model parameters.

\section{1) Load variability}

The continuous load variation in a power system can be considered as load uncertainty. The uncertainty of load in the system is typically modelled as normal distribution. The normal distribution parameters are mean $(\mu)$ and standard deviation $(\sigma)$. The level of load uncertainty considered in this study is $3 \sigma=5 \%$ of $\mu$. This level of load uncertainty represents typical load forecasting error over 24-hour time horizon [24].

2) System loading

A daily load profile presents the changes in system loading as per daily consumption pattern of customers. A typical daily loading pattern has been adopted in this study for a generic representation of load profile as low (40\%), base (60\%), intermediate (80\%), and peak (100\%) system loading [25].

\section{3) Load composition}

Power system loads at transmission level are aggregates of many different devices, which fall under the broad category of static and dynamic loads. The proportion of static and dynamic load in developed countries is typically within $30-40 \%$ and $60-70 \%$, respectively [26].

\section{4) Load model parameters}

Aggregated load models at a transmission level are best represented by a composite load model [26]. It can be represented through a combination of static (ZIP) and dynamic (induction machine, IM) models. It provides the most accurate representation of true system oscillation damping compared to other (i.e. full static or full dynamic) load models [27].

A conceptual representation of the composite load model is shown in Fig. 2(b) [28]. The ZIP and first order transfer function of an IM model is shown for real power in (8) and (9).

$$
\begin{gathered}
a\left(V / V_{0}\right)^{\alpha}+b\left(V / V_{0}\right)^{\beta}+c\left(V / V_{0}\right)^{\gamma} \\
\Delta P(s)=\left[\left(k_{p}+t_{p} s\right) /(1+T s)\right] \cdot \Delta v(s) \\
k_{p}=a \alpha+b \beta+c \gamma
\end{gathered}
$$

Composite load model parameters are constant impedance, current and power coefficients $(a, b, c)$, exponents $(\alpha, \beta, \gamma)$, voltage dependence index $\left(k_{p}\right)$ as defined in (10), voltage time constant $\left(t_{p}\right)$ and dynamic load time constant $(T)$. $V$ and $V_{0}$ are voltages after and before the disturbance, respectively [26]. Reactive power is defined similarly.

Load model parameters considered in this study are $\left[a=0.45, \quad b=-0.10, c=0.65, \alpha=0, \quad \beta=1, \gamma=2, t_{p}=76 s\right]$, as suggested by measurement based load modeling studies [29, 30]. Dynamic load time constant is the most demonstrative parameter of a load model [13]. Fig. 2(c) and (d) shows the $1^{\text {st }}$ order recovery of dynamic load with respect to different proportion of dynamic load and dynamic load time constants.

The value of dynamic load time constant used in this study is between $0.5 \mathrm{~s}$ and $20 \mathrm{~s}$, which coincides with the observation in [12] that most dynamic loads exhibit their dynamic behaviour within this range of time constants. Also, the timeframe of interest in small-disturbance stability studies is typically in the order of 10 to 20 seconds [16].

Though the first order dynamic model considered in this study has been identified as 'most widely used' generic dynamic load model in the literature, there are other more (a)

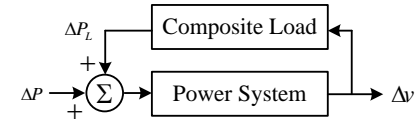

(b)

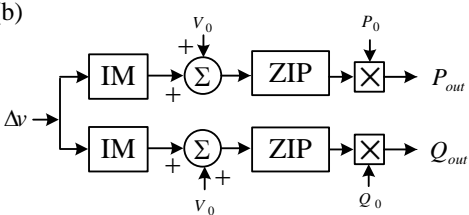

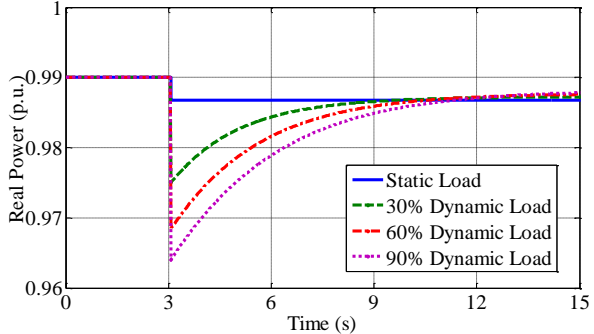

(c)

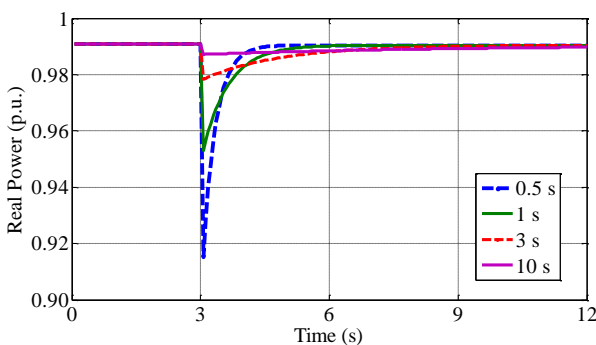

(d)

Fig. 2. Representation of (a) system-load interaction, (b) composite load model, and $1^{\text {st }}$ order recovery of dynamic load (c) with respect to different proportion of dynamic load, and (d) at different dynamic load time constants. 
comprehensive load models developed for specific power system studies [26]. The first order dynamic load model provides adequate results for small-disturbance stability studies and in cases where there are limited voltage variations in the system [31], hence it has been chosen for this study. The second-order and higher-order load models however, are better at capturing load behaviour during transients and would be more appropriate for large disturbance (transient) rotor angle stability analysis [26].

5) Location of load

The location of a load in the system can contribute to the system damping and risk-based small-disturbance security. However, locational issues are network and topology specific. The nearer a load to a generator is, the more influential it is on damping [15]. Location of load issue is not considered in this study, however it is acknowledged that future systems may witness greater spatial variability of loads, particularly as the penetration of electric vehicles increases.

\section{Test System AND PROBABILISTIC MODELLING}

The simulation network is a substantially modified version (with a high amount of renewable power penetration) of the NETS-NYPS test system (New England Test System - New York Power System) which has 5 areas, 16 machines and 68 buses, as shown in Fig. 3. Network data, component modelling and more information of this network are available in [32, 33]. Uncertain system variables considered are wind speed, load demand and solar irradiation, which follow normal, normal and beta distributions, respectively. Though Weibull distribution is used in the literature for modelling wind speed variation [34], wind speed can be also modelled using normal distribution, especially, when modelled for a shorter time intervals in the scale of an hour [35]. Normal distribution is considered for wind speed modelling in this research as the security assessment of a power system is studied over a short time window. The level of uncertainty considered here is $3 \sigma=10 \%$ of $\mu$, which is typical wind forecasting error over an hour time horizon [36].

Similarly, the probability distribution of load is typically considered in the literature as normal distribution [37], however, the future power systems may exhibit different distribution of load, particularly as the penetration of renewable resources, electric vehicles and demand-side participation increases [38]. In total, 49 uncertain parameters (35 loads, 7 wind and 7 solar farms) are modelled

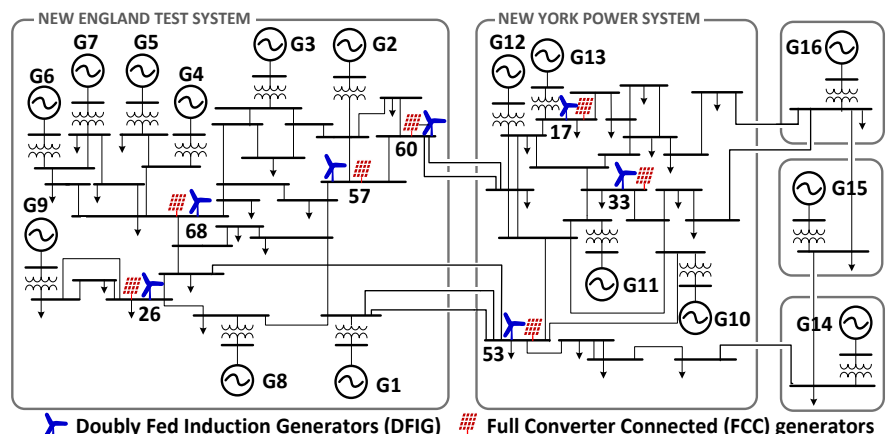

Fig. 3. Modified NETS-NYPS (New England Test System - New York Power System) network with a high amount of wind and solar generation. probabilistically. The locations of loads, DFIG wind and FCC (Full Converter Connected) solar are shown in Fig. 3.

In obtaining the system risk measures, a multi-level approach has been implemented consisting of the power flow in the network (by optimal power flow), small disturbance stability assessment (by modal analysis), and mapping critical mode damping into the risk contour (multiplying the severity function by the $p d f$ of critical mode damping). Probabilistic modelling of the input parameters and risk contours have been generated in MATLAB and OPF simulation has been solved using MATPOWER [39]. Modal analysis has been conducted in DIgSILENT PowerFactory.

The results have been obtained through the Monte Carlo simulation. A sufficient number of Monte Carlo run is determined through a stopping rule as in (11) [40].

$$
\varepsilon>\left[\left\{\Phi^{-1}(1-\delta / 2) \cdot \sqrt{\sigma^{2}(\mathbf{X}) / N}\right\} / \overline{\mathbf{X}}\right]
$$

In (11) $\varepsilon$ is the sample mean error, $\Phi^{-1}(\bullet)$ is the inverse Gaussian conditional probability distribution with a mean of zero and standard deviation of one, $\sigma^{2}(\bullet)$ is the variance of a sample, $\delta$ is the desired confidence level and $\overline{\mathbf{x}}$ is the mean of the samples. According to the criterion presented in (11), simulations can be stopped if the calculated sample mean error falls below a specific threshold, $\varepsilon$. It was identified that for this study 3500 samples results in $\varepsilon=0.2 \%$.

\section{RESULTS AND ANALYSIS}

The modal analysis and risk-assessment methods previously described have been executed on the NETS-NYPS test system to demonstrate the applicability of the proposed techniques.

\section{A. Probabilistic Security Boundary}

Fig. 4 shows the boxplots of the damping of critical eigenvalues to illustrate the relative influence of load attributes on small-disturbance stability studies. Damping of the critical eigenvalues have been presented with respect to the change in load uncertainty, system loading, proportion of dynamic load, and dynamic load time constant. The range of different load attributes corresponds to the discussion and references as presented in III.A.

When one of the load attributes of interest is changing, all others remain fixed at their base values in each case of Fig. 4 $(\mathrm{a}-\mathrm{d})$. The base case simulation has been considered as $80 \%$ system loading, 50\% dynamic load, 5\% load uncertainty and 3 s dynamic load time constant.

Each boxplot consists of $3500 \mathrm{MC}$ simulations. The boxplot with whisker shows the range of variation of critical mode damping around a specific operating point. The line inside the box is the median value, while edge of the box shows upper $(75 \%)$ and lower $(25 \%)$ quartile of eigenvalue dispersion. The spacing inside the box indicates the degree of dispersion and skewness of eigenvalues. The whiskers correspond to $95 \%$ coverage of eigenvalues.

The contribution of load forecasting error on the oscillation damping can be observed from Fig. 4(a). The distinct feature of this curve is the spread of the eigenvalues over the complex plane. The eigenvalue dispersion widens with an increased level of load uncertainty and it is approaching the instability 
limit. With a further increase in load uncertainty, the right margin of the boxplot would probably cross the instability boundary. It is not only the median value but also the range of eigenvalues that is important for assessing the system security.

The impact of system loading can be clearly observed from Fig. 4(b). Two distinct features of the curve can be seen clearly. One is the wider spread of the eigenvalues over the complex plane with the increase of the system loading. Another is the movement of the curve towards right to the unstable region with the increase of the system loading in the network. Interpolating between the cases studied, it appears that the system starts to experience instability (with $95 \%$ confidence) at around $85 \%$ system loading.

A similar trend can be observed for the proportion of dynamic load and dynamic load time constant in 4(c) and 4(d), respectively. Again interpolating, it can be estimated that the system could exhibit instability when the proportion of dynamic load and dynamic load time constant are higher than $72 \%$ and time constant smaller than $2.7 \mathrm{~s}$, respectively.

The system is highly sensitive to the dynamic load time constant. As the dynamic load time constant decreases, the system stability rapidly deteriorates. As seen when testing the other load attributes, as the system moves towards the unstable region, the eigenvalue spread increases.

\section{B. Value at Risk (VaR)}

The probabilistic metric VaR can be introduced to identify the value of specific load attributes to estimate a stable operating limit. VaRs for different load attributes can be obtained from Fig. 4. Load uncertainty VaR cannot be determined as the uncertainty level does not exceed the stable security limit within the simulation range.

The VaR related to the system loading is $85 \%$ at $95 \%$ confidence interval, which means that there is a probability of the system being unstable if load exceeds $85 \%$, provided that all other parameters remain at their base value (i.e. 50\% dynamic load, 5\% load uncertainty and 3s dynamic load time constant). Similarly, the VaR associated with the proportion of

(a)

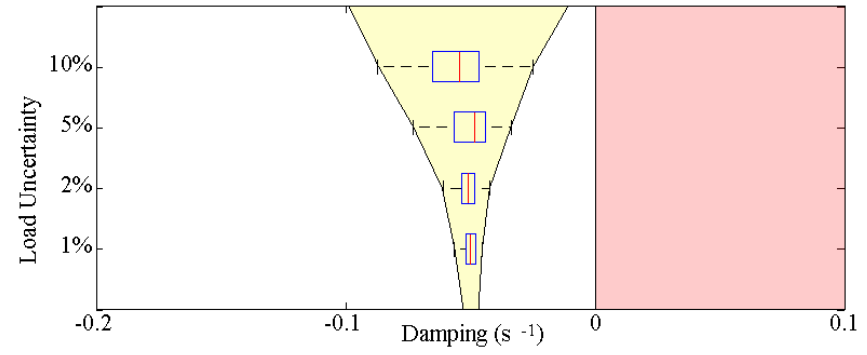

(c)

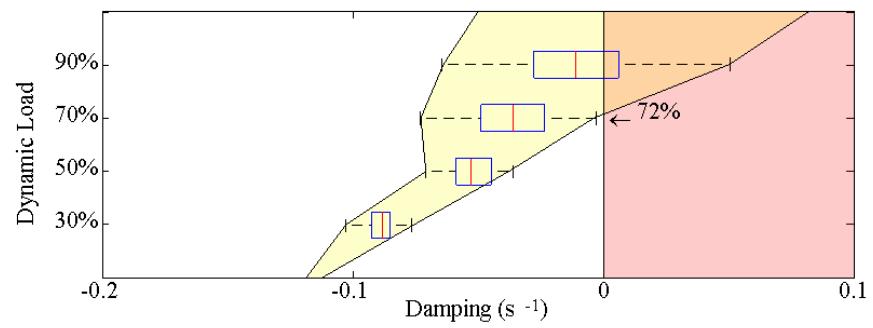

dynamic load and dynamic load time constant is $72 \%$ and 2.7s, respectively (Fig. 4).

\section{Risk-based Small-Disturbance Security Margin}

Fig. 5 shows the iso-risk contours considering the influence of different levels of load uncertainty to the proportion of dynamic load, system loading profile and dynamic load time constant. These risk values have been obtained using the severity function previously described with $r$ and $m$ equal to 10 and 10 respectively. The values of $r$ and $m$ have no real significance. They are merely to express the relative risk and can be normalized. The lightly shaded areas in the left side of the graphs show lower risk levels i.e. secure operating region. As the proportion of load attributes increase, the shaded area becomes darker portraying higher levels of risk. It can be seen from the figure that there is a general trend of a slight increase in the risk with the increment of uncertainty. The dashed lines within the figure represent the levels of risk (with $r=10$ and $m=10$ ). It can be seen from Fig. 5 that a change in dynamic load time constant has a more significant impact on the risk level than a change in the proportion of dynamic load or system loading.

The risk levels associated with the interactions among prominent load attributes are presented in Fig. 6 (with $r$ and $m$ equal to 10 and 10 respectively). There is a low level of risk when system operates at $72 \%$ (or lower) proportion of dynamic load and $85 \%$ (or less) system loading. As the proportion of dynamic load and system loading increase from these values the risk-contour shades become darker showing higher levels of risk. The same trend can be seen from the contour plots of dynamic load vs. dynamic load time constant and system loading vs. dynamic load time constant. The darker shade in the risk-contour appears at approximately $2.7 \mathrm{~s}$ time (which is the VaR limit) for dynamic load time constant.

The significance of these results lies in the representation of risk-based security margin, which has some inherent advantages, as below:

- The iso-risk contours portray the same levels of risk at (b)

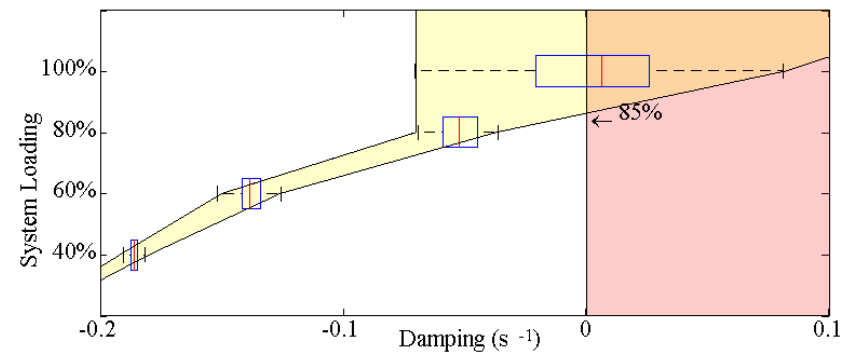

(d)

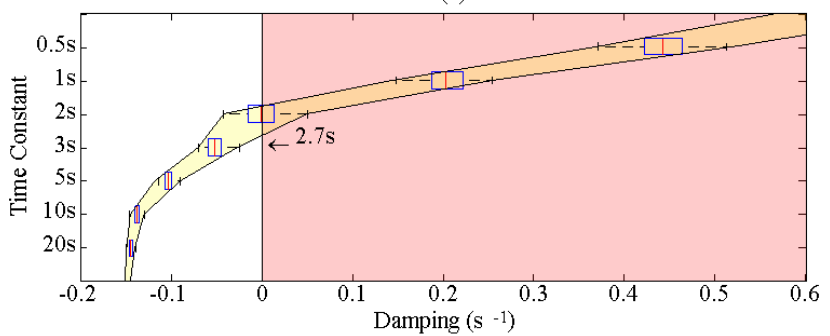

Fig. 4. Boxplot of critical eigenvalues considering the impact of load attributes on small-disturbance stability studies. Damping of the critical eigenvalue with respect to the change in (a) load uncertainty, (b) system loading, (c) proportion of dynamic load, and (d) dynamic load time constant. 
different operating conditions with a combination of different sets of parameters. It also shows the risk variation through the operating region.

- The risk profile explores the relative contribution of system parameters at varying levels of risk. More influential parameters can be identified in this way by looking at the changes of risk levels that they cause.

- A gradual increase in risk can be seen as the system moves towards severe operating conditions. Hence, it provides an indication when the system approaches to instability.

- It shows the combined weighting of low-impact lowprobability, low-impact high-probability, high-impact low-probability, and high-impact high-probability situations. Hence, it reflects the total composite risk, and not simply that from the worst case scenario.

- Consideration of a wide range of scenarios through uncertainty explores the level of risk involved in future uncertain conditions.

Though the application of severity function has been demonstrated on a small-disturbance stability analysis, where modal analysis clearly distinguishes the stable and unstable operating region, the same concept can be easily mapped through to voltage (and frequency) stability studies where for example the system overvoltage (or over-frequency) and under-voltage (or under-frequency) boundaries can be used. In case of voltage stability assessment the pdf of loadability indices can be produced based on continuation power flow at system critical bus. These can be then multiplied by the developed semi-continuous function (where the discontinuity will be at the point of voltage collapse) to map through to the risk profile. Similarly, for frequency stability, the pdf of frequency nadir will be generated and multiplied by the developed severity function, where the discontinuity will be at the load-shedding frequency point.

\section{Impact of the Shape of the Severity Function}

The influence of the shape of the severity function has been considered by choosing an exponential function (instead of a linear function) in the unstable region and corresponding system risk profile is shown in Fig. 7. The illustrative result has been presented considering different levels of dynamic load and system loading. Fig. 7(a) shows the risk profile with the piece-wise linear monotonic severity function (with severity multiplier $m=10$, and intercept ratio $r=10$ ), whereas Fig. 7(b) presents the risk profile with an exponential function in the unstable region, $y=e^{100 x}+y_{2}^{x 0}\left(y_{2}^{x 0}\right.$ is the $y$-axis intercept of unstable region as shown in Fig. 1). With an exponential increase of severity in the unstable region (as in Fig. 7(b)), increasingly unstable cases are more heavily weighted and the risk-contour clearly distinguishes stable and unstable
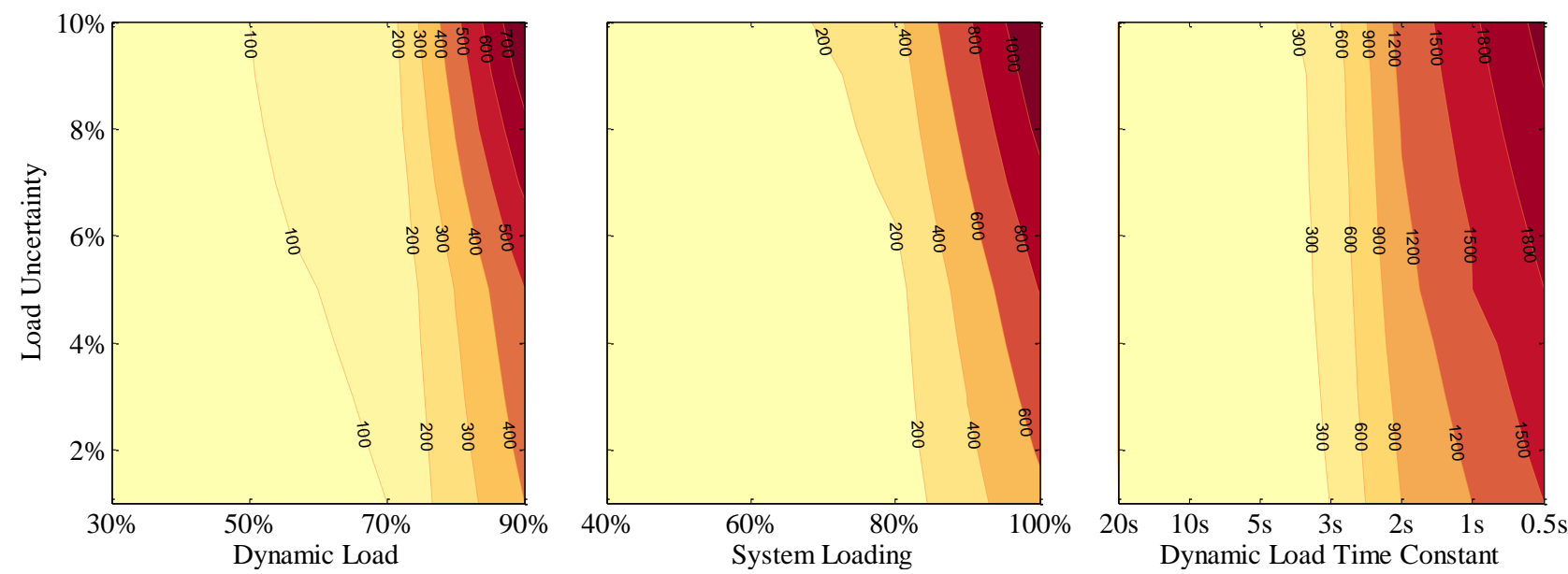

Fig. 5. Risk-contour considering the impact of dynamic load, system loading, and dynamic load time constant at different levels of load uncertainty.
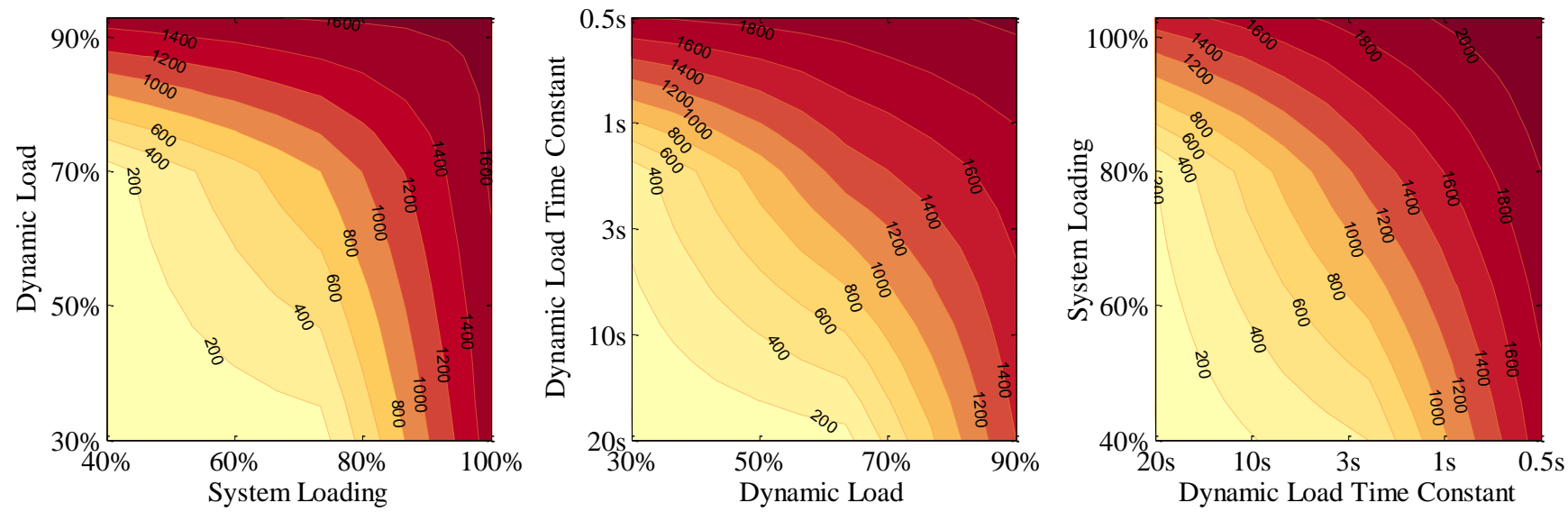

Fig. 6. Risk-contour with dynamic load vs. system loading, dynamic load vs. dynamic load time constant, and system loading vs. dynamic load time constant. 
operating range. Whilst such a severity curve could be considered, it is possible to capture risk similarly using the more simply defined piece-wise linear function. Fig. 7(c) shows the risk profile with the piece-wise linear severity function (with $m=10, r=10000$ ). This setting of the severity function also heavily weights any instability. As can be seen, the resulting risk profile is extremely similar to that produced using the exponential severity curve in Fig. 7(b). Hence, by appropriate selection of the severity multiplier $(m)$ and intercept ratio $(r)$, the severity of the system instability can be modelled according to the user's specification. The next Section presents a detailed sensitivity analysis of the parameters of the severity function.

\section{E. Sensitivity of the Severity Function}

The impact of the parameters of the severity function on the system risk profile is presented in Fig. 8. The incurred levels of risk for dynamic load vs. system loading have been presented as an illustration considering nine sets of values for severity multiplier $(m)$ and intercept ratio $(r)$. The impact of increasing intercept ratio can be seen while moving from left to right, and the impact of increasing severity multiplier while moving from top to bottom in Fig. 8. With a low value of $r$, the difference between stable and unstable cases is not clearly weighted in the risk profile. This represents a very gentle risk gradient in the top left risk-contour, which does not appropriately differentiate between cases which include stable and unstable conditions. As $r$ increases (moving towards right along Fig. 8), the unstable cases are more heavily weighted compared to stable cases, and hence the risk-contour clearly distinguishes stable and unstable operating range.

When the value of $r$ is small, such as the leftmost plots in Fig. 8, increasing the value of $m$ can contribute slightly to increase the relative risk of conditions with unstable operation. However, it can be seen clearly from Fig. 8 that the impact of $m$ is very small compared to the impact of $r$. This impact (a) $\mathrm{m}=10, \mathrm{r}=10$

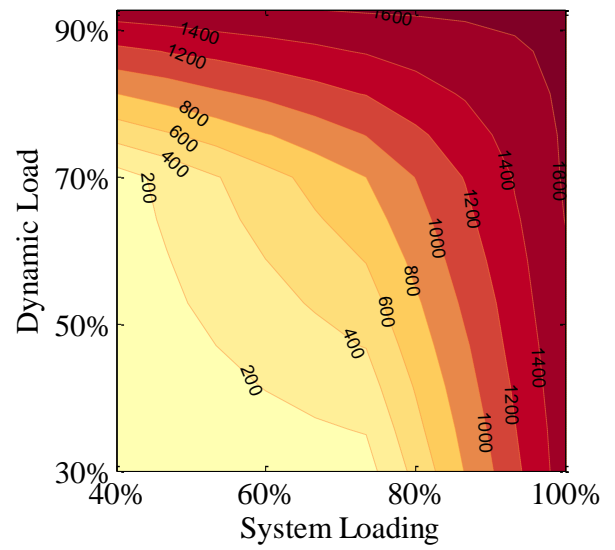

(b) Exponential function in unstable region

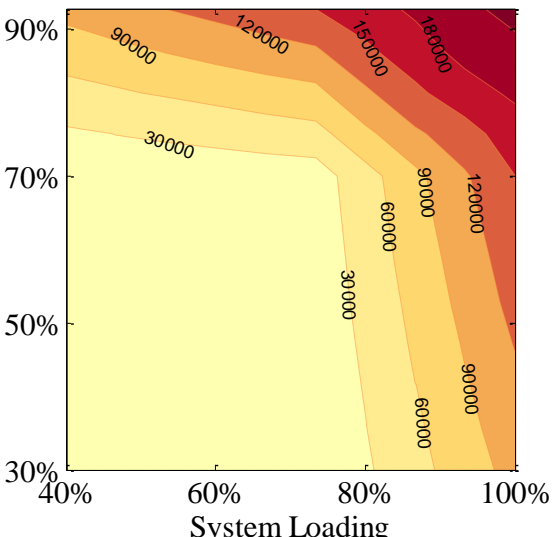

System Loading

Fig. 7. Risk-contour with different shapes of the severity functions for dynamic load vs. system loading.
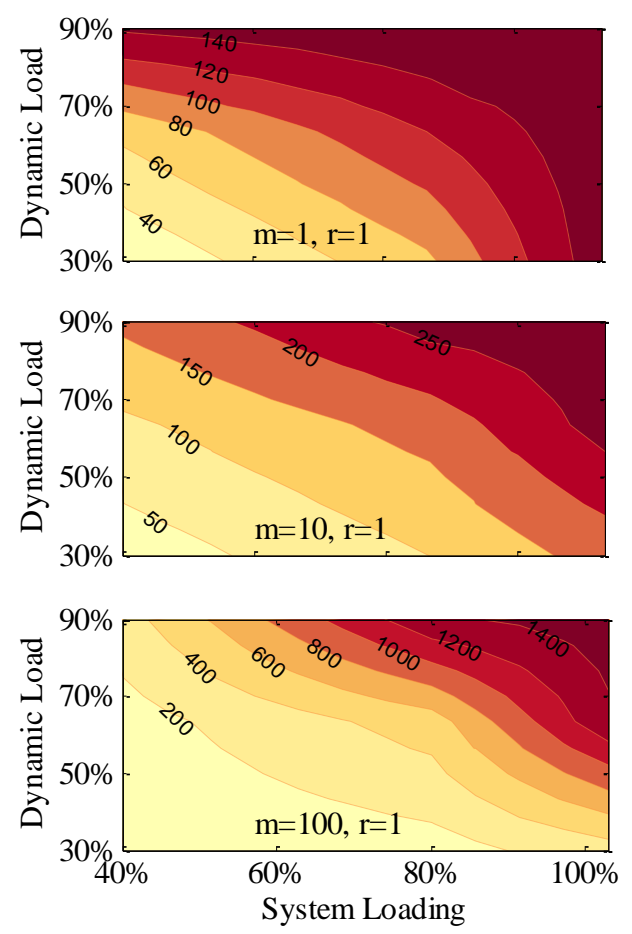
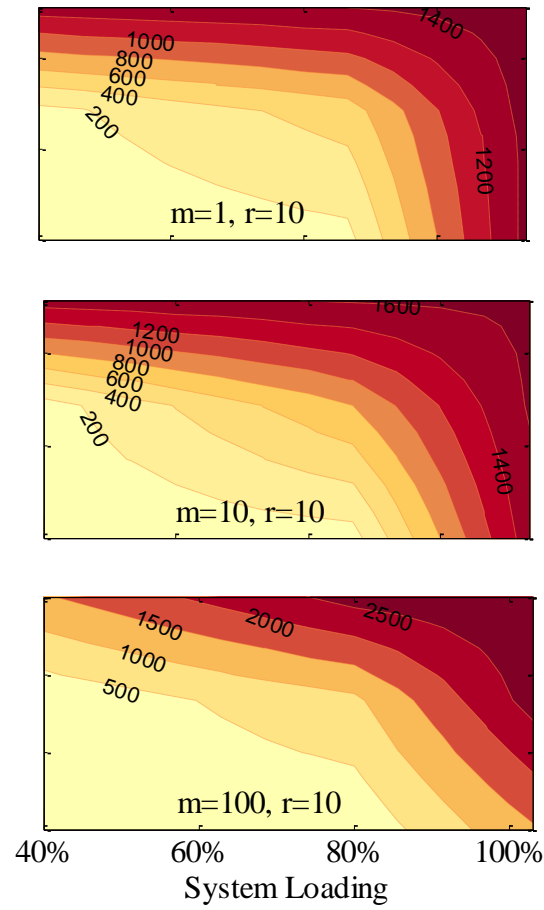

(c) $m=10, r=10000$

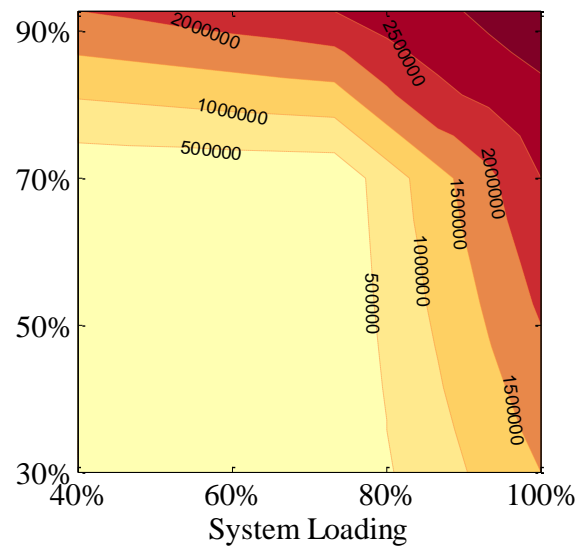

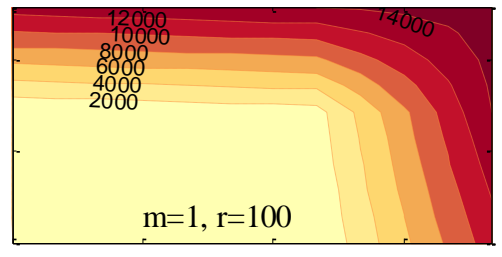
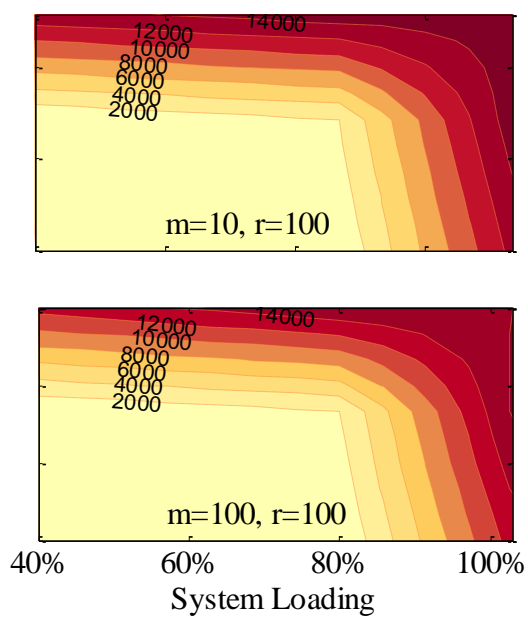

Fig. 8. Risk-contour of dynamic load vs. system loading with respect to alternative severity functions. 
further diminishes as the value of $r$ increases, with almost no difference seen in the rightmost plots of Fig. 8 when $r$ is 100 .

These parameters of the severity function will need to be tuned by operators depending on how risk is perceived/ avoided in their systems. If they have an abundance of controllable devices they can use to restore stability then their attitude toward marginal instability will be very different to a transmission system operator (TSO) who has no controllable devices and who wants to maintain a good distance from the instability boundary. For the first case, they may set $r$ quite low as they can bring the system back from instability with relative ease, whereas the second TSO will set $r$ extremely high in order to penalize all cases that have even the slightest chance of instability as they will not be able to correct this in an operational timeframe.

\section{F. A High-Risk Scenario with Violation of Multidimensional Security Margin}

The security margin for system stability is projected from the risk profile of the system as $85 \%, 72 \%$ and $2.7 \mathrm{~s}$, for system loading, proportion of dynamic load, and dynamic load time constant respectively. Once the security margin for individual load attribute is known, it is less likely that the system operates close to that operating limit. However, if certain conditions of the network bring the system operation close to the security margin from all dimensions (such as when the system loading, proportion of dynamic load and dynamic load time constant are $85 \%, 72 \%$ and $2.7 \mathrm{~s}$, respectively), Fig. 9 shows how severe that incident can be. Fig. 9 shows the eigenvalue plot with 5\% load uncertainty when abovementioned three load attributes stretch to their security margin. The probability of instability in such a stressed condition of the network could be as high as $49 \%$. This is recognizing the severity of violation of the security limit identified through the risk-based small-disturbance stability analysis and presented through the iso-risk contours.

It would be possible to develop a multi-dimensional risk plane that could encapsulate the risk as a function of these multiple stresses at once. However, such a hyperplane would be impossible to visualize effectively without flattening it in specific dimensions (effectively resulting in the risk-contours previously produced).

\section{CONCLUSIONS}

The risk-profile of a power system with the consideration of load attributes has been obtained in this study through smalldisturbance security analysis. The risk profiling methodology quantifies the impact (severity) and probability of certain operating conditions and how the risk profile is affected by various uncertainties and system parameters. The severity function proposed and implemented within this work distinctly sets apart the associated severity levels for oscillatory stable and unstable scenarios.

The eigenvalue measure through boxplot over the complex plane with respect to load attributes captures the trend of the direction of the eigenvalue movement (towards instability). This further facilitates identification of the probabilistic security boundary for variety of load attributes considered. The security boundary explores the maximum level of different load attributes which can be achieved before there is

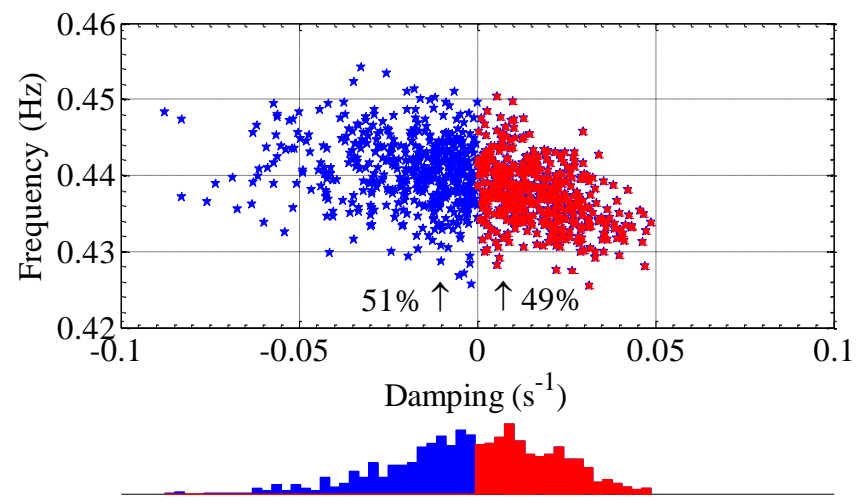

Fig. 9. Illustrative eigenvalue plot of a highly stressed network, which meets the boundary of multi-dimensional security margin through the system loading, proportion of dynamic load, and dynamic load time constant.

a known probability that the system will lose oscillatory stability.

The iso-risk contour shows different operating conditions which maintain the same risk levels and gradually increasing risk level caused by the subsequent changes in operating conditions. The dynamic system behavior is captured and incorporated in the risk-based security assessment. The riskcontours provide a guidance to observe the risk level at particular operating conditions and the change in risk levels as a function of system load attributes.

The risk-based security margins capture the properly weighted severity in operating conditions. The results show the relative significance of different load attributes, where dynamic load time constant is the most influential parameter. This further establishes the importance of load modelling and accurately knowing its important parameters.

The system risk profile is highly influenced by the impact of load dynamics, which is becoming an area of interest with the emergence of new types of loads in modern power systems. This study provides a guideline for determining the flexibility of system operation with respect to load attributes and system uncertainties. Given the high stressed conditions observed in many systems today, the risk-based approach will serve better in this type of environment.

The value of the risk is typically, and should be, expressed in terms of monetary values, however, in this particular case that is not possible yet due to the lack of a financial quantification (costing) of risk of power system instability. The costing or the value of power system (in)stability is an ongoing area of research.

\section{REFERENCES}

[1] W. Li, "Introduction," in Risk Assessment of Power Systems:Models, Methods, and Applications, ed: Wiley-IEEE Press, 2014, pp. 1-560.

[2] N. Ming, J. D. McCalley, V. Vittal, and T. Tayyib, "Online risk-based security assessment," IEEE Trans. Power Syst., vol. 18, pp. 258-265, 2003.

[3] J. McCalley, S. Asgarpoor, L. Bertling, R. Billinion, H. Chao, J. Chen, J. Endrenyi, R. Fletcher, A. Ford, C. Grigg, G. Hamoud, D. Logan, A. P. Meliopoulos, N. R. M. Ni, L. Salvaderi, M. Schilling, Y. Schlumberger, A. Schneider, and C. Singh, "Probabilistic security assessment for power system operations," presented at the IEEE PES Gen Meeting, 2004.

[4] M. Negnevitsky, N. Dinh Hieu, and M. Piekutowski, "Risk Assessment for Power System Operation Planning With High Wind Power Penetration," IEEE Trans. Power Syst., vol. 30, pp. 1359-1368, 2015. 
[5] A. Dissanayaka, U. D. Annakkage, B. Jayasekara, and B. Bagen, "RiskBased Dynamic Security Assessment," IEEE Trans. Power Syst., vol. 26, pp. 1302-1308, 2011.

[6] C. K. Pans, Z. Y. Dong, P. Zhang, and X. Yin, "Probabilistic analysis of power system small signal stability region," in Intl. Conf. on Control and Automation, 2005, pp. 503-509 Vol. 1.

[7] R. Preece and J. V. Milanovic, "Risk-Based Small-Disturbance Security Assessment of Power Systems," IEEE Trans. Power Delivery, vol. 30, pp. 590-598, 2015.

[8] R. Arrieta, M. A. Rios, and A. Torres, "Contingency Analysis and Risk Assessment of Small Signal Instability," in IEEE Power Tech, 2007, pp. 1741-1746.

[9] J. L. Rueda, D. G. Colome, and I. Erlich, "Assessment and Enhancement of Small Signal Stability Considering Uncertainties," IEEE Trans. Power Syst., vol. 24, pp. 198-207, 2009.

[10] J. L. Rueda and I. Erlich, "Probabilistic framework for risk analysis of power system small-signal stability," Proc. of the Inst. of Mech. Engrs, Part O: Journal of Risk and Reliability, vol. 226, pp. 118-133, 2012.

[11] R. Preece and J. V. Milanovic, "Assessing the risk of small disturbance instability in mixed AC/DC networks," in Bulk Pow. Syst. Dynamics and Control - IREP, 2013, pp. 1-8.

[12] I. A. Hiskens and J. V. Milanovic, "Load modelling in studies of power system damping," IEEE Trans. Power Syst., vol. 10, pp. 1781-1788, 1995.

[13] J. V. Milanovic and I. A. Hiskens, "Effects of load dynamics on power system damping," IEEE Trans. Power Syst., vol. 10, pp. 1022-1028, 1995

[14] CIGRE_Working_Group, "Review of the Current Status of Tools and Techniques for Risk-Based and Probabilistic Planning in Power Systems," C4.601 on Power System Security Assessment, 2010.

[15] Y. Mishra, Z. Y. Dong, J. Ma, and D. J. Hill, "Induction motor load impact on power system eigenvalue sensitivity analysis," IET Gen., Trans. \& Dist., vol. 3, pp. 690-700, 2009.

[16] P. Kundur, J. Paserba, V. Ajjarapu, G. Andersson, A. Bose, C. Canizares, N. Hatziargyriou, D. Hill, A. Stankovic, C. Taylor, T. Van Cutsem, and V. Vittal, "Definition and classification of power system stability IEEE/CIGRE joint task force on stability terms and definitions," IEEE Trans. Power Syst., vol. 19, pp. 1387-1401, 2004.

[17] K. N. Hasan, R. Preece, and J. V. Milanovic, "Priority Ranking of Critical Uncertainties Affecting Small-Disturbance Stability Using Sensitivity Analysis Techniques," IEEE Trans Power Syst., vol. PP, pp. $1-1,2016$.

[18] K. N. Hasan, R. Preece, and J. V. Milanović, "Efficient identification of critical parameters affecting the small-disturbance stability of power systems with variable uncertainty," in 2016 IEEE PESGM, 2016, pp. 15.

[19] "Load representation for dynamic performance analysis [of power systems]," IEEE Trans. Power Syst. , vol. 8, pp. 472-482, 1993.

[20] D. J. Hill, "Nonlinear dynamic load models with recovery for voltage stability studies," IEEE Trans Power Syst., vol. 8, pp. 166-176, 1993.

[21] D. Karlsson and D. J. Hill, "Modelling and identification of nonlinear dynamic loads in power systems," IEEE Trans Power Syst., vol. 9, pp. 157-166, 1994

[22] J. V. Milanovic, K. Yamashita, S. M. Villanueva, S. Ž. Djokic, and L. M. Korunović, "International Industry Practice on Power System Load Modeling," IEEE Trans Power Syst., vol. 28, pp. 3038-3046, 2013.

[23] H. Renmu, J. Ma, and D. J. Hill, "Composite load modeling via measurement approach," IEEE Trans Power Syst., vol. 21, pp. 663-672, 2006.

[24] J. W. Taylor, "Short-Term Electricity Demand Forecasting Using Double Seasonal Exponential Smoothing," The Journal of the Operational Research Society, vol. 54, pp. 799-805, 2003.

[25] National_Grid, "Future Energy Scenarios: UK gas and electricity transmission," July 2015.

[26] CIGRE_Working_Group_C4.605, "Modelling and aggregation of loads in flexible power networks, Jovica V. Milanović, (Convenor), Julija Matevosiyan, Anish Gaikwad, Alberto Borghetti, Saša Ž. Djokić, Zhao Yang Dong, Andrew Halley, Lidija M. Korunović, Sergio Martinez Villanueva, Jin Ma, Pouyan Pourbeik, Fernanda Resende, Stefan Sterpu, Fortunato Villella, Koji Yamashita, Odin Auer, Karim Karoui, Dimitry Kosterev, Shu Kwan Leung, Dumisani Mtolo, Samila Mat Zali, Adam Collin, Yizheng Xu, ISBN: 978-2-85873-261-6," 2014.

[27] K. Wen-Shiow, "The effect of load models on unstable low-frequency oscillation damping in Taipower system experience w/wo power system stabilizers," IEEE Trans. Power Syst., vol. 16, pp. 463-472, 2001.
[28] DIGSILENT_PowerFactory, "Technical Reference. Accessed Jan. 2016. available; http://www.digsilent.de/index.php/downloads.html."

[29] EPRI, "Measurement-based load modeling. [available online]: http://www.epri.com/abstracts/Pages/ProductAbstract.aspx?ProductId=0 $00000000001014402, " 2006$

[30] I. R. Navarro, "Dynamic Load Models for Power Systems," PhD Thesis, Lund University, Sweden, 2002.

[31] A. Borghetti, R. Caldon, and C. A. Nucci, "Generic dynamic load models in long-term voltage stability studies," International Journal of Electrical Power \& Energy Systems, vol. 22, pp. 291-301, 2000.

[32] G. Rogers, Power System Oscillations: Norwell: Kluwer Academic Publishers, 2000.

[33] B. Pal and B. Chaudhuri, Robust Control in Power Systems. New York: Springer Inc., 2005.

[34] S. Q. Bu, W. Du, H. F. Wang, Z. Chen, L. Y. Xiao, and H. F. Li, "Probabilistic Analysis of Small-Signal Stability of Large-Scale Power Systems as Affected by Penetration of Wind Generation," IEEE Trans Power Syst., vol. 27, pp. 762-770, 2012.

[35] W. Zhou, Y. Peng, and H. Sun, "Optimal wind-thermal coordination dispatch based on risk reserve constraints," European Transactions on Electrical Power, vol. 21, pp. 740-756, 2011.

[36] G. Giebel, R. Brownsword, and G. Kariniotakis, "The State-Of-The-Art in Short-Term Prediction of Wind Power: A Literature Overview. ANEMOS," 2012.

[37] V. A. Evangelopoulos and P. S. Georgilakis, "Optimal distributed generation placement under uncertainties based on point estimate method embedded genetic algorithm," Generation, Transmission \& Distribution, IET, vol. 8, pp. 389-400, 2014.

[38] H. Marzooghi, G. Verbič, and D. J. Hill, "Aggregated demand response modelling for future grid scenarios," Sustainable Energy, Grids and Networks, vol. 5, pp. 94-104, 2016.

[39] R. D. Zimmerman, S. Murillo, x, C. E. nchez, and R. J. Thomas, "MATPOWER: Steady-State Operations, Planning, and Analysis Tools for Power Systems Research and Education," IEEE Trans. Power Syst., vol. 26, pp. 12-19, 2011.

[40] R. Preece and J. V. Milanovic, "Efficient Estimation of the Probability of Small-Disturbance Instability of Large Uncertain Power Systems," IEEE Trans. Power Syst., vol. 31, pp. 1063-1072, 2016.

Kazi N. Hasan (GS'08-M'14) is working as a Post-doctoral research associate at the University of Manchester, UK. His research interest includes analysing the impact of distributed energy resources and new types of loads into the electricity grid.

Robin Preece (GS'10, M'13) received his BEng degree in Electrical and Electronic Engineering in 2009 and his $\mathrm{PhD}$ degree in 2013, both from the University of Manchester, United Kingdom. He is currently a Lecturer of Power Systems Engineering at the same institution.

Jovica V. Milanović (M'95, SM'98, F'10) received the Dipl.Ing. and M.Sc. degrees from the University of Belgrade, Belgrade, Yugoslavia, the Ph.D. degree from the University of Newcastle, Newcastle, Australia, and the Higher Doctorate (D.Sc. degree) from The University of Manchester, U.K., all in electrical engineering. Currently, he is a Professor of Electrical Power Engineering, Deputy Head of School and Director of External Affairs in the School of Electrical and Electronic Engineering at the University of Manchester, U.K., Visiting Professor at the University of Novi Sad, Serbia, University of Belgrade, Serbia and Conjoint Professor at the University of Newcastle, Australia. Professor Milanovic is a Chartered Engineer in the UK, Foreign member of the Serbian Academy of Engineering Sciences, Fellow of the IET, Fellow of the IEEE, Distinguished IEEE PES Lecturer and currently serves on IEEE PES Governing Board as Regional Representative for Europe, Middle East and Africa and on the IEEE PES Fellows Committee. 